\title{
Distinguishing Fuzzballs from Black Holes through Their Multipolar Structure
}

\author{
Massimo Bianchi® ${ }^{1}$ Dario Consoli $\odot,{ }^{2}$ Alfredo Grillo $\odot,{ }^{1}$ Josè Francisco Morales $\odot,{ }^{1}$ \\ Paolo Pani $\odot,{ }^{3}$ and Guilherme Raposo ${ }^{3}$ \\ ${ }^{1}$ Dipartimento di Fisica, Universit di Roma "Tor Vergata" and Sezione INFN Roma2, \\ Via della ricerca scientifica 1, 00133, Roma, Italy \\ ${ }^{2}$ Mathematical Physics Group, University of Vienna, Boltzmanngasse 51090 Vienna, Austria \\ ${ }^{3}$ Dipartimento di Fisica, "Sapienza" Università di Roma and Sezione INFN Romal, \\ Piazzale Aldo Moro 5, 00185, Roma, Italy
}

(Received 7 July 2020; accepted 19 October 2020; published 24 November 2020)

\begin{abstract}
Within general relativity, the unique stationary solution of an isolated black hole is the Kerr spacetime, which has a peculiar multipolar structure depending only on its mass and spin. We develop a general method to extract the multipole moments of arbitrary stationary spacetimes and apply it to a large family of horizonless microstate geometries. The latter can break the axial and equatorial symmetry of the Kerr metric and have a much richer multipolar structure, which provides a portal to constrain fuzzball models phenomenologically. We find numerical evidence that all multipole moments are typically larger (in absolute value) than those of a Kerr black hole with the same mass and spin. Current measurements of the quadrupole moment of black-hole candidates could place only mild constraints on fuzzballs, while future gravitational-wave detections of extreme mass-ratio inspirals with the space mission LISA will improve these bounds by orders of magnitude.
\end{abstract}

DOI: 10.1103/PhysRevLett.125.221601

Introduction.-Owing to the black-hole $(\mathrm{BH})$ uniqueness and no-hair theorems [1,2] (see also Refs. [3-5]), within general relativity (GR) any stationary BH in isolation is also axisymmetric and its multipole moments satisfy an elegant relation [6]

$$
\mathcal{M}_{\ell}^{\mathrm{BH}}+i \mathcal{S}_{\ell}^{\mathrm{BH}}=\mathcal{M}^{\ell+1}(i \chi)^{\ell},
$$

where $\mathcal{M}_{\ell}\left(\mathcal{S}_{\ell}\right)$ are the Geroch-Hansen mass (current) multipole moments [6,7], the suffix "BH" refers to the $\mathrm{BH}$ metric, $\mathcal{M}=\mathcal{M}_{0}$ is the mass, $\chi \equiv \mathcal{J} / \mathcal{M}^{2}$ is the dimensionless spin, and $\mathcal{J}=\mathcal{S}_{1}$ is the angular momentum (we use natural units throughout). (For a generic spacetime, the multipole moments of order $\ell$ are rank- $\ell$ tensors, which reduce to scalar quantities $\mathcal{M}_{\ell}$ and $\mathcal{S}_{\ell}$ in the axisymmetric case. See below for the general definition.) Equation (1) implies that all Kerr moments with $\ell \geq 2$ can be written only in terms of the mass $\mathcal{M}$ and angular momentum $\mathcal{J}$ of the spacetime. Introducing the dimensionless quantities $\overline{\mathcal{M}}_{\ell} \equiv \mathcal{M}_{\ell} / \mathcal{M}^{\ell+1}$ and $\overline{\mathcal{S}}_{\ell} \equiv \mathcal{S}_{\ell} / \mathcal{M}^{\ell+1}$, the nonvanishing moments are

$$
\overline{\mathcal{M}}_{2 n}^{\mathrm{BH}}=(-1)^{n} \chi^{2 n}, \quad \overline{\mathcal{S}}_{2 n+1}^{\mathrm{BH}}=(-1)^{n} \chi^{2 n+1},
$$

Published by the American Physical Society under the terms of the Creative Commons Attribution 4.0 International license. Further distribution of this work must maintain attribution to the author(s) and the published article's title, journal citation, and DOI. Funded by SCOAP ${ }^{3}$. for $n=0,1,2, \ldots$. The fact that $\mathcal{M}_{\ell}=0\left(\mathcal{S}_{\ell}=0\right)$ when $\ell$ is odd (even) is a consequence of the equatorial symmetry of the Kerr metric. Likewise, the fact that all multipoles with $\ell \geq 2$ are proportional to (powers of) the spin-as well as their specific spin dependence-is a peculiarity of the Kerr metric, that is lost for other compact-object solutions in GR [8,9] and also for BH solutions in other gravitational theories.

Testing whether these properties hold for an astrophysical dark object provides an opportunity to perform multiple null-hypothesis tests of the Kerr metric-for example, by measuring independently three multipole moments such as the mass, spin, and mass quadrupole $\mathcal{M}_{2}$ - serving as a genuine strong-gravity test of Einstein's gravity [10-16], along with other proposed observational tests of fuzzballs (see, e.g., Refs. [17,18]). In this context, it is intriguing that current gravitational-wave (GW) observations (especially the recent GW190814 [19] and GW190521 [20,21]) do not exclude the existence of exotic compact objects other than $\mathrm{BHs}$ and neutron stars.

In GR, BHs have curvature singularities that are conjectured to be always covered by event horizons [22-24]. At the quantum level, BHs behave as thermodynamical systems with the area of the event horizon and its surface gravity playing the role of the entropy and temperature, respectively $[25,26]$. In fact, a $\mathrm{BH}$ can evaporate emitting Hawking radiation [27]. This gives rise to a number of paradoxes that can be addressed in a consistent quantum theory of gravity such as string theory [28]. 
For special classes of extremal [charged Bogomol' nyi-Prasad-Sommerfield (BPS)] BHs [29-31] one can precisely count the microstates that account for the BH entropy. In some cases, one can even identify smooth horizonless geometries with the same mass, charges, and angular momentum as the corresponding $\mathrm{BH}$. These geometries represent some of the microstates in the low-energy (super)gravity description. The existence of a nontrivial structure at the putative horizon scale is the essence of the fuzzball proposal [32-35]. In the latter, many properties of BHs in GR emerge from an averaging procedure over a large number of microstates, or as a "collective behavior" of fuzzballs [36-40]. So far it has been hard to find a statistically significant fraction of microstate geometries both for fivedimensional (three-charge) and for four-dimensional (fourcharge) BPS BHs. Yet, several classes of solutions based on a multicenter ansatz [41-46] have been found and their string theory origin uncovered [47-49].

Although in viable astrophysical scenarios $\mathrm{BHs}$ are expected to be neutral, charged BPS BHs are a useful toy model to explore the properties of their microstates. Extending the fuzzball proposal to neutral, non-BPS BHs in four dimensions and finding predictions that can be observationally tested so as to distinguish this from other proposals and from the standard BH picture in GR [16] remain an open challenge.

In this Letter and in a companion paper [50], we investigate the differences in the multipolar structure between $\mathrm{BHs}$ and fuzzballs. As we shall argue, already at the level of the quadrupole moments the nonaxisymmetric geometry of generic microstates in the four-dimensional fuzzball model leads to a much richer phenomenology and to potentially detectable deviations from GR.

Setup.-Our method is based on Thorne's seminal work on the multipole moments of a stationary isolated object [51]. The idea is to choose a suitable coordinate system-so-called asymptotically Cartesian mass centered (ACMC)-whereby the mass and current multipole moments can be directly extracted from a multipolar expansion of the metric components. In an ACMC system, the metric of a stationary asymptotically flat object can be written as [50]

$d s^{2}=-\left(1-c_{00}\right) d t^{2}+c_{0 i} d t d x_{i}+\left(1+c_{00}\right) d x_{i}^{2}+\cdots$

with $x_{i}=\{x, y, z\}$, and $c_{00}$ and $c_{0 i}$ admitting a sphericalharmonic expansion of the form [51]

$$
\begin{aligned}
& c_{00}=2 \sum_{\ell=0}^{\infty} \sum_{m=-\ell}^{\ell} \frac{1}{r^{1+\ell}} \sqrt{\frac{4 \pi}{2 \ell+1}}\left(\mathcal{M}_{\ell m} Y_{\ell m}+\ell^{\prime}<\ell\right), \\
& c_{0 i}=2 \sum_{\ell=1}^{\infty} \sum_{m=-\ell}^{\ell} \frac{1}{r^{1+\ell}} \sqrt{\frac{4 \pi(\ell+1)}{\ell(2 \ell+1)}}\left(\mathcal{S}_{\ell m} Y_{i, \ell m}^{B}+\ell^{\prime}<\ell\right),
\end{aligned}
$$

in terms of the scalar $\left(Y_{l m}\right)$ and axial vector $\left(Y_{i, \ell m}^{B}\right)$ spherical harmonics. [It can be shown that the radial
$\left(Y_{i, \ell m}^{R}\right)$ and electric $\left(Y_{i, \ell m}^{E}\right)$ vector spherical harmonics only appear in subleading terms and do not affect the multipole moments [51].] The expansion coefficients $\mathcal{M}_{\ell m}$ and $\mathcal{S}_{\ell m}$ are the mass and current multipole moments of the spacetime, respectively. They can be conveniently packed into a single complex harmonic function

$$
H=\sum_{\ell=0}^{\infty} \sum_{m=-\ell}^{\ell} \frac{1}{r^{1+\ell}} \sqrt{\frac{4 \pi}{2 \ell+1}}\left(\mathcal{M}_{\ell m}+i \mathcal{S}_{\ell m}\right) Y_{\ell m} .
$$

In the case of the Kerr metric, $H$ is simply given by

$$
H_{\mathrm{Kerr}}=\frac{\mathcal{M}}{\sqrt{x_{1}^{2}+x_{2}^{2}+\left(x_{3}-i \frac{\mathcal{J}}{\mathcal{M}}\right)^{2}}},
$$

with two centers at positions $z= \pm \mathcal{J} / \mathcal{M}$ along the $z$ axis. The harmonic expansion of Eq. (6) does not contain $m \neq 0$ terms, so that for each $\ell$ the moment tensors reduce to the scalars $\mathcal{M}_{\ell} \equiv \mathcal{M}_{\ell 0}$ and $\mathcal{S}_{\ell} \equiv \mathcal{S}_{\ell 0}$. The same holds for more general axisymmetric metrics. [The normalization of Thorne's multipoles can be chosen in order to correspond to the Geroch-Hansen ones [6,7] used in Eq. (1) in the axisymmetric case [52].]

Here we consider fuzzball solutions of gravity in four dimensions minimally coupled to four Maxwell fields and three complex scalars. A general class of extremal solutions of the Einstein-Maxwell system is described by a metric of the form [53-55]

$$
d s^{2}=-e^{2 U}(d t+w)^{2}+e^{-2 U} \sum_{i=1}^{3} d x_{i}^{2},
$$

with

$$
\begin{aligned}
e^{-4 U}= & L_{1} L_{2} L_{3} V-K^{1} K^{2} K^{3} M+\frac{1}{2} \sum_{I>J}^{3} K^{I} K^{J} L_{I} L_{J} \\
& -\frac{M V}{2} \sum_{I=1}^{3} K^{I} L_{I}-\frac{1}{4} M^{2} V^{2}-\frac{1}{4} \sum_{I=1}^{3}\left(K^{I} L_{I}\right)^{2}, \\
*_{3} d w= & \frac{1}{2}\left(V d M-M d V+K^{I} d L_{I}-L_{I} d K^{I}\right),
\end{aligned}
$$

where $*_{3}$ is the Hodge dual in three-dimensional flat space, $\left\{V, L_{I}, K^{I}, M\right\}$ are eight harmonic functions associated with the four electric and four magnetic charges, and $I, J=1,2,3$.

Fuzzball solutions are obtained by distributing the charges of the eight harmonic functions among $N$ centers in such a way that the geometry near each center lifts to a regular five-dimensional geometry. More explicitly, we take 


$$
\begin{array}{rlrl}
V & =v_{0}+\sum_{a=1}^{N} \frac{v_{a}}{r_{a}}, & M & =m_{0}+\sum_{a=1}^{N} \frac{m_{a}}{r_{a}}, \\
K^{I}=k_{0}^{I}+\sum_{a=1}^{N} \frac{k_{a}^{I}}{r_{a}}, & L_{I}=\ell_{I, 0}+\sum_{a=1}^{N} \frac{\ell_{I, a}}{r_{a}},
\end{array}
$$

with $r_{a}=\left|\mathbf{x}-\mathbf{x}_{a}\right|$ the distance from the $a$ th center.

Results. - Comparing the metric (7) with the definition of an ACMC metric (3), one can extract the multipole moments of the fuzzball solution (details are given in Ref. [50]). The fuzzball multipole moments are encoded in the multipole harmonic function

$$
H=\frac{1}{4} \sum_{a=1}^{N}\left[V+i M+\sum_{I=1}^{3}\left(L_{I}-i K^{I}\right)\right] .
$$

This complex harmonic function is a generalization of the Kerr case [Eq. (6)]; the latter can be interpreted as a twocenter solution, with the Schwarzschild case corresponding to a single center. The above expression is instead valid for generic $\mathrm{N}$-center solutions, regardless of the presence of electromagnetic and scalar fields.

Expanding the harmonic function $H$ yields the multipole moments

$$
\begin{aligned}
\mathcal{M}_{\ell m} & =\frac{1}{4} \sum_{a=1}^{N}\left(v_{a}+\sum_{I} \ell_{I, a}\right) R_{\ell m}^{a}, \quad \ell \geq 0, \\
\mathcal{S}_{\ell m} & =\frac{1}{4} \sum_{a=1}^{N}\left(m_{a}-\sum_{I} k_{a}^{I}\right) R_{\ell m}^{a}, \quad \ell \geq 1,
\end{aligned}
$$

with $\mathcal{M}_{00}=\mathcal{M}$ and

$$
R_{\ell m}^{a}=\left|\mathbf{x}_{a}\right|^{\ell} \sqrt{\frac{4 \pi}{2 \ell+1}} Y_{\ell m}^{*}\left(\theta_{a}, \phi_{a}\right)
$$

As in the case of axisymmetric geometries, we define dimensionless moments

$$
\overline{\mathcal{M}}_{\ell m}=\frac{\mathcal{M}_{\ell m}}{\mathcal{M}^{\ell+1}}, \quad \overline{\mathcal{S}}_{\ell m}=\frac{\mathcal{S}_{\ell m}}{\mathcal{M}^{\ell+1}} .
$$

We center the coordinate system in the center of mass and orient the $z$ axis along the angular momentum, so that

$$
\begin{aligned}
\frac{1}{4} \sum_{a=1}^{N}\left(v_{a}+\sum_{I} \ell_{I, a}\right) & \mathbf{x}_{a}=0 \\
\frac{1}{4} \sum_{a=1}^{N}\left(m_{a}-\sum_{I} k_{a}^{I}\right) & \mathbf{x}_{a}=\mathcal{J} \mathbf{e}_{z},
\end{aligned}
$$

with $\mathbf{e}_{z}$ the unit vector along $z$. With this choice $\mathcal{M}_{1 m}=0$, $\mathcal{S}_{1, \pm 1}=0$, and $\mathcal{S}_{10}=\mathcal{J}$.
Equations (11) are one of our main results, as they allow us to compute the multipole moments of any multicenter microstate geometry. In fact, our method can be straightforwardly applied to any metric in ACMC form. In the following, we will focus on some specific cases.

Examples.-The simplest horizonless geometries arise from three-center solutions. We consider fuzzballs that asymptote to $\mathrm{BHs}$ carrying three electric $\left(Q_{I}\right)$ and one magnetic $\left(P_{0}\right)$ charge, obtained from orthogonal branes, so we require that $K^{I}$ and $M$ vanish at order $1 / r$. Up to a reordering of the centers, the general solution can be written in the form [45]

$$
\begin{aligned}
V & =1+\sum_{a=1}^{3} \frac{1}{r_{a}}, \quad M=\kappa_{1} \kappa_{2} \kappa_{3} \kappa_{4}\left(\frac{1}{r_{1}}-\frac{1}{r_{2}}\right), \\
L_{1} & =1+\kappa_{4}\left(\frac{\kappa_{3}}{r_{1}}-\frac{\kappa_{2}}{r_{2}}\right), \quad L_{2}=1+\kappa_{1} \kappa_{4}\left(\frac{\kappa_{3}}{r_{2}}-\frac{\kappa_{2}}{r_{1}}\right), \\
L_{3} & =1+\kappa_{1}\left(\frac{\kappa_{2} \kappa_{3}}{r_{1}}+\frac{\kappa_{2} \kappa_{3}}{r_{2}}+\frac{\left(\kappa_{2}+\kappa_{3}\right)^{2}}{r_{3}}\right), \\
K_{1} & =\kappa_{1}\left(-\frac{\kappa_{2}}{r_{1}}-\frac{\kappa_{3}}{r_{2}}+\frac{\kappa_{2}+\kappa_{3}}{r_{3}}\right), \\
K_{2} & =\frac{\kappa_{3}}{r_{1}}+\frac{\kappa_{2}}{r_{2}}-\frac{\kappa_{2}+\kappa_{3}}{r_{3}}, \quad K_{3}=\kappa_{4}\left(\frac{1}{r_{2}}-\frac{1}{r_{1}}\right),
\end{aligned}
$$

with $\kappa_{\alpha}$ some arbitrary integers.

Regular solutions describe microstates of a (nonrotating) BPS BH with mass

$$
\mathcal{M}=\frac{1}{4}\left(Q_{1}+Q_{2}+Q_{3}+P_{0}\right)
$$

and charges

$$
\begin{aligned}
& Q_{1}=\kappa_{4}\left(\kappa_{3}-\kappa_{2}\right), \quad Q_{2}=\kappa_{1} \kappa_{4}\left(\kappa_{3}-\kappa_{2}\right), \\
& Q_{3}=\kappa_{1}\left(\kappa_{2}^{2}+4 \kappa_{2} \kappa_{3}+\kappa_{3}^{2}\right), \quad P_{0}=3 .
\end{aligned}
$$

Besides the integer parameters $\kappa_{\alpha}$, the solution depends on some continuous parameters, namely, the distances between the centers $r_{a b}=\left|\mathbf{x}_{a}-\mathbf{x}_{b}\right|$. These are constrained by the so-called "bubble equations" [53], ensuring regularity of the five-dimensional lift and absence of closed timelike curves. In the three-center case, one has

$$
\begin{aligned}
& r_{12}=\frac{2 \kappa_{1} \kappa_{4}\left(\kappa_{2}-\kappa_{3}\right)^{2} r_{23}}{\kappa_{1} \kappa_{4}\left(2 \kappa_{2}^{2}+5 \kappa_{2} \kappa_{3}+2 \kappa_{3}^{2}\right)+\left[\kappa_{2}+\kappa_{4}-\kappa_{1} \kappa_{3}\left(1-\kappa_{2} \kappa_{4}\right)\right] r_{23}}, \\
& r_{13}=\frac{\kappa_{1} \kappa_{4}\left(2 \kappa_{2}+\kappa_{3}\right)\left(\kappa_{2}+2 \kappa_{3}\right) r_{23}}{\kappa_{1} \kappa_{4}\left(2 \kappa_{2}^{2}+5 \kappa_{2} \kappa_{3}+2 \kappa_{3}^{2}\right)-\left(\kappa_{1}-1\right)\left(\kappa_{2}+\kappa_{3}\right) r_{23}},
\end{aligned}
$$

which allow one to express $r_{12}$ and $r_{13}$ in terms of $r_{23}=L$, the surviving continuous parameter ("modulus") labeling the microstate. Asymptotically the solution coincides with 
TABLE I. The first dimensionless multipole moments of some representative three-center microstate geometries in the $k^{2} \gg L$. Moments with $m<0$ follow from $\overline{\mathcal{M}}_{\ell,-m}=(-1)^{m} \overline{\mathcal{M}}_{\ell, m}^{*}$.

\begin{tabular}{lcccccccc}
\hline \hline Solution & $\left(\kappa_{1}, \kappa_{2}, \kappa_{3}, \kappa_{4}\right)$ & $\overline{\mathcal{S}}_{10}$ & $\overline{\mathcal{M}}_{20}$ & $\overline{\mathcal{M}}_{21}$ & $\overline{\mathcal{M}}_{22}$ & $\overline{\mathcal{S}}_{20}$ & $\overline{\mathcal{S}}_{21}$ & $\overline{\mathcal{S}}_{22}$ \\
\hline $\mathbf{A}$ & $(1,0, k, k)$ & 0 & $8 / 27 k^{4}$ & 0 & 0 & 0 & 0 & 0 \\
B & $(1,0,1, k)$ & $L / k$ & $L^{2} / k^{2}$ & 0 & $3 \sqrt{3} L^{2} / 2 \sqrt{2} k^{3}$ & 0 & $-3 L^{2} / \sqrt{2} k^{3}$ & 0 \\
C & $(3,0, k, 2 k)$ & $4 \sqrt{3} L / 11^{2} k^{3}$ & $144 L^{2} / 11^{4} k^{4}$ & $72 \sqrt{2} L^{2} / 11^{4} k^{4}$ & $72 \sqrt{6} L^{2} / 11^{4} k^{4}$ & $-164 L^{2} / 11^{4} k^{5}$ & $-48 \sqrt{2} L^{2} / 11^{4} k^{3}$ & $2 \sqrt{6} L^{2} / 11^{3} k^{3}$ \\
Kerr-Newman & & $\chi$ & $-\chi^{2}$ & 0 & 0 & 0 & 0 & 0 \\
\hline \hline
\end{tabular}

the Kerr-Newman metric [56], whose multipolar structure is the same [57] as in the Kerr case [see Eq. (1)].

A summary of the first multipole moments for some representative cases is shown in Table I. The general expressions for the multipole moments are cumbersome, so we present them in the limit of large mass $\left(\kappa_{\alpha} \gg 1\right)$, which is also the most interesting one from a phenomenological point of view, since it corresponds to objects with mass arbitrarily larger than the Planck mass. We consider three representative arrangements of the three centers:

Case (A) Equilateral triangle. $\left(\kappa_{1}, \kappa_{2}, \kappa_{3}, \kappa_{4}\right)=$ $(1,0, k, k)$. These microstate geometries fall into the class of "scaling solutions" characterized by zero angular momentum, $\mathcal{J}=0$, equal charges $\vec{Q}=\left(k^{2}, k^{2}, k^{2}\right)$, and mass $\mathcal{M}=\frac{3}{4}\left(1+k^{2}\right)$. Thanks to $\mathbf{Z}_{3}$ symmetry around $z$, the nontrivial mass multipole moments read

$$
\mathcal{M}_{2 p+3 n, 3 n}=\mathcal{M}(-L)^{2 p+3 n} \frac{\sqrt{(2 p+6 n) !(2 p) !}}{2^{2 p+3 n}(p+3 n) ! n !}
$$

where $L=r_{12}=r_{23}=r_{31}$. Thus, at variance with the Kerr case, the mass quadrupole moments are not spin induced; they can be nonzero even if the spin $\mathcal{J}$ vanishes. Furthermore, for $\ell \geq 3$ they also have $m \neq 0$ components of the mass moments. The large $k$ limit of all quadrupole moments are displayed in Table I.

Case (B) Isosceles triangle. $\left(\kappa_{1}, \kappa_{2}, \kappa_{3}, \kappa_{4}\right)=(1,0,1, k)$. These microstate geometries possess nonvanishing angular momentum, $\mathcal{J}=\{(k-1) k L / 2[k(L+2)-L]\}$, charges $\vec{Q}=(k, k, 1)$, and mass $\mathcal{M}=[(2+k) / 2]$. In this case $L=r_{23}=r_{31}>r_{12}$. For $k \rightarrow \infty$ and $L \ll 1$ (see Table I), the multiple moments coincide with those of the Kerr metric modulo the factors $(-1)^{n}$ in Eq. (2). In particular, while the Kerr metric is oblate $\left(\mathcal{M}_{2}<0\right)$, these solutions are prolate $\left(\mathcal{M}_{2}>0\right)$. However, for finite values of $k$, the solution also displays quadrupole moments that break axial symmetry, e.g., $\mathcal{M}_{22}$ and $\mathcal{S}_{21}$.

Case (C) Scalene triangle. $\left(\kappa_{1}, \kappa_{2}, \kappa_{3}, \kappa_{4}\right)=(3,0, k, 2 k)$. These microstate geometries possess a nonvanishing angular momentum $\mathcal{J}$, which is a complicated function of $k$ and $L$, with $L=r_{23}<r_{12}<r_{31}$, charges $\vec{Q}=\left(2 k^{2}, 6 k^{2}, 3 k^{2}\right)$, and mass $\mathcal{M}=\left[\left(3+11 k^{2}\right) / 4\right]$. For large $k$, one finds $\mathcal{J} \sim$ $[(\sqrt{3} k L) / 4]$. Triangle inequalities require $\nu \equiv\left(L / 12 k^{2}\right)<$ $1-(1 / \sqrt{2})$. The multipole moments for large $k$ are displayed in Table I. In this case, both the axisymmetry and the equatorial symmetry of the Kerr metric are broken, as shown by the fact that the multipole moments $\mathcal{M}_{\ell m}$ and $\mathcal{S}_{\ell m}$ are generically nonzero.

It is interesting to observe that the mass and current multipole moments of these microstate geometries are typically larger than those of a Kerr-Newman BH with same mass and angular momentum. A representative example of this property is shown in Fig. 1, where we display some ratios between multipole moments of microstate geometries of type $\mathrm{C}$ and those of a Kerr $\mathrm{BH}$. We focus on the quadratic invariants

$$
\operatorname{tr} \mathcal{M}_{\ell}^{2}=\sum_{m=-\ell}^{\ell}\left|\mathcal{M}_{\ell m}\right|^{2}, \quad \operatorname{tr} S_{\ell}^{2}=\sum_{m=-\ell}^{\ell}\left|S_{\ell m}\right|^{2} .
$$

We have explored numerically a large region of the whole $\left(\kappa_{\alpha}, L\right)$ parameter space and found that quadratic invariants for the microstate geometries are typically bigger than those of Kerr BHs for any $\ell$ [50]. It would be interesting to find a general proof of this property, which is analogous to the fact that the Lyapunov exponent of unstable null geodesics

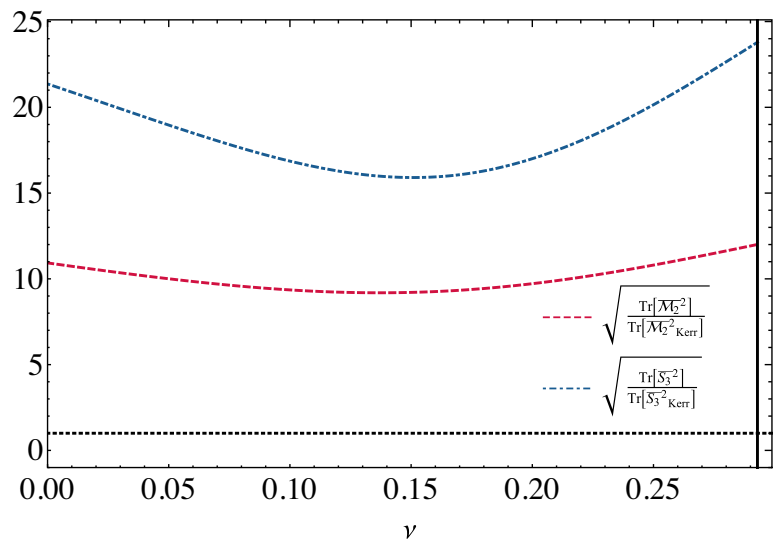

FIG. 1. Ratios between the quadratic invariants for the first multipole moments of a fuzzball (solution $\mathbf{C}$ ) and a Kerr BH with the same angular momentum, as a function of $\nu=L /\left(12 k^{2}\right)$ with $k=1$. The vertical solid line corresponds to the upper bound $\nu_{\max }=1-1 / \sqrt{2}$. The horizontal dotted black line refers to the fuzzball and Kerr moments being identical. The fuzzball moments are larger than the corresponding Kerr ones, which is a typical property [50]. 
near the photon sphere is maximum for the $\mathrm{BH}$ solution [40]. In other words, both for the multipole moments and for the Lyapunov exponent, the BH solution appears to be an extremum point in the space of the solutions.

Phenomenological implications.-The above examples are representatives of some general features of this large family of solutions. In particular, the $\ell \geq 2$ multipole moments of fuzzball geometries are not necessarily spin induced as in the Kerr case, they can break axial and equatorial symmetries and are larger than in the Kerr case. The peculiar multipolar structure and the striking deviation from the Kerr multipoles provides a portal to constrain fuzzball models with current and future observations, with both electromagnetic and GW probes [16].

By analyzing the accretion flow near the supermassive BH in M87, the Event Horizon Telescope placed a mild bound on its dimensionless (axisymmetric) quadrupole moment, $\left|\overline{\mathcal{M}}_{2}-\overline{\mathcal{M}}_{2}^{\mathrm{BH}}\right| \lesssim 4$ [58]. Furthermore, in a coalescence the quadrupole moment of the binary components affect the GW signal through a next-to-next-to-leading post-Newtonian correction $[59,60]$. Constraints on parametrized post-Newtonian deviations using the events from the first LIGO-Virgo Catalog $[61,62]$ can be mapped into a constraint $\left|\overline{\mathcal{M}}_{2}-\overline{\mathcal{M}}_{2}^{\mathrm{BH}}\right| \lesssim 1$, in particular, using the events GW151226 and GW170608 [63]. Comparing with deviations found in the microstate solutions, current bounds are not particularly stringent.

While current GW constraints will become slightly more stringent in the next years as the sensitivity of the groundbased detectors improve [64], much tighter bounds will come from extreme mass-ratio inspirals (EMRIs), one of the main targets of the future space mission LISA [65]. Although EMRI data analysis is challenging [66-69], the potential reward is unique: a detection of these systems can be used to measure the ( $m=0$, mass) quadrupole moment $\overline{\mathcal{M}}_{2}$ of the central supermassive object with an accuracy of one part in $10^{4}[66,70]$, offering unprecedented tests of exotic compact objects $[9,71,72]$.

While our results suggest that very strong constraints on fuzzball geometries can be set with EMRIs, a precise analysis requires a class of neutral, nonextremal solutions, which would further imply the absence of extra emission channels (e.g., dipolar radiation). For astrophysically viable objects, we expect that the multipolar structure is the only discriminant with respect to the Kerr BH case, which can be explored with the methods presented here.

In addition to having a different quadrupole moment, microstate geometries are much less symmetric than the Kerr metric, which implies the existence of multipole moments that are identically zero in the Kerr case (see also Refs. [9,73]). Investigating how multipole moments that break equatorial symmetry or axisymmetry (e.g., $\mathcal{S}_{2 m}$ and $\mathcal{M}_{2 m}$ with $m \neq 0$ ) affect the GW waveform and their phenomenological consequences is an important topic that is left for a follow-up work.
Finally, a broad statistical analysis shows that certain invariant combinations of the $\ell \geq 2$ multipole moments of three-center microstate geometries are larger than those of the corresponding Kerr $\mathrm{BH}$ in a wide region of the fourdimensional parameter space and are always larger than their corresponding value in the $L \rightarrow 0$ limit [50]. If confirmed, this result would imply that any future measurement of the invariant combinations of the multipole moments smaller than the $\mathrm{BH}$ ones can potentially rule out this family of solutions to be typical microstates of the corresponding $\mathrm{BH}$, with important consequences for the fuzzball scenario.

D. C. was supported by FWF Austrian Science Fund via the SAP P30531-N27. P. P. acknowledges financial support provided under the European Union's H2020 ERC, Starting Grant Agreement No. DarkGRA-757480 and under the Italian Ministry of Education, University and Research (MIUR) PRIN and FARE programmes (GWNEXT, CUP: B84I20000100001), and support from the Amaldi Research Center funded by the MIUR program "Dipartimento di Eccellenza" (CUP: B81I18001170001).

Note added.-Recently, a related work by Iosif Bena and Daniel R. Mayerson appeared [74] (see also the more recent companion [75]). The idea and aims of that paper are similar to ours. Reference [74] focuses on axisymmetric geometries in the $\mathrm{BH}$ limit, whereas our results are valid beyond axial symmetry in regions where the microstate geometries can significantly deviate from the $\mathrm{BH}$ metric.

[1] B. Carter, Axisymmetric Black Hole Has Only Two Degrees of Freedom, Phys. Rev. Lett. 26, 331 (1971).

[2] S. Hawking and G. Ellis, The Large Scale Structure of Space-Time, Cambridge Monographs on Mathematical Physics (Cambridge University Press, Cambridge, England, 2011).

[3] M. Heusler, Stationary black holes: Uniqueness and beyond, Living Rev. Relativity 1, 6(1998).

[4] P. T. Chrusciel, J. L. Costa, and M. Heusler, Stationary black holes: Uniqueness and beyond, Living Rev. Relativity 15, 7 (2012).

[5] D. Robinson, Four Decades of Black Holes Uniqueness Theorems (Cambridge University Press, Cambridge, England, 2009).

[6] R. Hansen, Multipole moments of stationary space-times, J. Math. Phys. (N.Y.) 15, 46 (1974).

[7] R. P. Geroch, Multipole moments. II. Curved space, J. Math. Phys. (N.Y.) 11, 2580 (1970).

[8] P. Pani, I-Love-Q relations for gravastars and the approach to the black-hole limit, Phys. Rev. D 92, 124030 (2015); Erratum, Phys. Rev. D 95, 049902 (2017).

[9] G. Raposo, P. Pani, and R. Emparan, Exotic compact objects with soft hair, Phys. Rev. D 99, 104050 (2019).

[10] D. Psaltis, Probes and tests of strong-field gravity with observations in the electromagnetic spectrum, Living Rev. Relativity 11, 9 (2008). 
[11] J. R. Gair, M. Vallisneri, S. L. Larson, and J. G. Baker, Testing general relativity with low-frequency, space-based gravitational-wave detectors, Living Rev. Relativity 16, 7 (2013).

[12] N. Yunes and X. Siemens, Gravitational-wave tests of general relativity with ground-based detectors and pulsar timing-arrays, Living Rev. Relativity 16, 9 (2013).

[13] E. Berti et al., Testing general relativity with present and future astrophysical observations, Classical Quantum Gravity 32, 243001 (2015).

[14] V. Cardoso and L. Gualtieri, Testing the black hole no-hair hypothesis, Classical Quantum Gravity 33, 174001 (2016).

[15] L. Barack et al., Black holes, gravitational waves and fundamental physics: A roadmap, Classical Quantum Gravity 36, 143001 (2019).

[16] V. Cardoso and P. Pani, Testing the nature of dark compact objects: A status report, Living Rev. Relativity 22, 4 (2019).

[17] T. Hertog and J. Hartle, Observational implications of Fuzzball formation, Gen. Relativ. Gravit. 52, 67 (2020).

[18] B. Guo, S. Hampton, and S. D. Mathur, Can we observe fuzzballs or firewalls?, J. High Energy Phys. 07 (2018) 162.

[19] R. Abbott et al. (LIGO Scientific and Virgo Collaborations), GW190814: Gravitational waves from the coalescence of a 23 solar mass black hole with a 2.6 solar mass compact object, Astrophys. J. 896, L44 (2020).

[20] R. Abbott et al. (LIGO Scientific and Virgo Collaborations), GW190521: A Binary Black Hole Merger with a Total Mass of $150 M_{\odot}$, Phys. Rev. Lett. 125, 101102 (2020).

[21] R. Abbott et al. (LIGO Scientific and Virgo Collaborations), Properties and astrophysical implications of the 150 Msun binary black hole merger GW190521, Astrophys. J. Lett. 900, L13 (2020).

[22] R. Penrose, Gravitational collapse: The role of general relativity, Riv. Nuovo Cimento 1, 252 (1969); Gen. Relativ. Gravit. 34, 1141 (2002).

[23] R. M. Wald, Gravitational collapse and cosmic censorship, in Black Holes, Gravitational Radiation and the Universe: Essays in Honor of C.V. Vishveshwara (1997), pp. 69-85. [arXiv:gr-qc/9710068].

[24] R. Penrose, Singularities of Spacetime, in Theoretical Principles in Astrophysics and Relativity (Chicago University Press, Chicago, 1978), p. 217.

[25] J. D. Bekenstein, Black holes and entropy, Phys. Rev. D 7, 2333 (1973).

[26] S. W. Hawking, Black holes and thermodynamics, Phys. Rev. D 13, 191 (1976).

[27] S. Hawking, Particle creation by black holes, Commun. Math. Phys. 43, 199 (1975); Erratum, Commun. Math. Phys. 46, 206 (1976).

[28] S. D. Mathur, The information paradox: A pedagogical introduction, Classical Quantum Gravity 26, 224001 (2009).

[29] A. Strominger and C. Vafa, Microscopic origin of the Bekenstein-Hawking entropy, Phys. Lett. B 379, 99 (1996).

[30] G. T. Horowitz, J. M. Maldacena, and A. Strominger, Nonextremal black hole microstates and U duality, Phys. Lett. B 383, 151 (1996).

[31] J. M. Maldacena, A. Strominger, and E. Witten, Black hole entropy in $M$ theory, J. High Energy Phys. 12 (1997) 002 .
[32] O. Lunin and S.D. Mathur, AdS/CFT duality and the black hole information paradox, Nucl. Phys. B623, 342 (2002).

[33] O. Lunin and S. D. Mathur, Statistical Interpretation of Bekenstein Entropy for Systems with a Stretched Horizon, Phys. Rev. Lett. 88, 211303 (2002).

[34] S. D. Mathur, The fuzzball proposal for black holes: An elementary review, Fortschr. Phys. 53, 793 (2005).

[35] S. D. Mathur, Fuzzballs and the information paradox: A summary and conjectures, arXiv:0810.4525.

[36] M. Bianchi, D. Consoli, and J. Morales, Probing fuzzballs with particles, waves and strings, J. High Energy Phys. 06 (2018) 157.

[37] M. Bianchi, D. Consoli, A. Grillo, and J. F. Morales, The dark side of fuzzball geometries, J. High Energy Phys. 05 (2019) 126.

[38] I. Bena, E. J. Martinec, R. Walker, and N. P. Warner, Early scrambling and capped BTZ geometries, J. High Energy Phys. 04 (2019) 126.

[39] I. Bena, P. Heidmann, R. Monten, and N. P. Warner, Thermal decay without information loss in horizonless microstate geometries, SciPost Phys. 7, 063 (2019).

[40] M. Bianchi, A. Grillo, and J. F. Morales, Chaos at the rim of black hole and fuzzball shadows, J. High Energy Phys. 05 (2020) 078.

[41] I. Bena, S. Giusto, R. Russo, M. Shigemori, and N. P. Warner, Habemus Superstratum! A constructive proof of the existence of superstrata, J. High Energy Phys. 05 (2015) 110.

[42] I. Bena, E. Martinec, D. Turton, and N. P. Warner, Momentum fractionation on superstrata, J. High Energy Phys. 05 (2016) 064.

[43] I. Bena, S. Giusto, E. J. Martinec, R. Russo, M. Shigemori, D. Turton, and N. P. Warner, Smooth Horizonless Geometries Deep Inside the Black-Hole Regime, Phys. Rev. Lett. 117, 201601 (2016)

[44] I. Bena, S. Giusto, E. J. Martinec, R. Russo, M. Shigemori, D. Turton, and N. P. Warner, Asymptotically-flat supergravity solutions deep inside the black-hole regime, J. High Energy Phys. 02 (2018) 014.

[45] M. Bianchi, J. F. Morales, L. Pieri, and N. Zinnato, More on microstate geometries of 4d black holes, J. High Energy Phys. 05 (2017) 147.

[46] I. Bena, D. Turton, R. Walker, and N. P. Warner, Integrability and black-hole microstate geometries, J. High Energy Phys. 11 (2017) 021.

[47] S. Giusto, J. F. Morales, and R. Russo, D1D5 microstate geometries from string amplitudes, J. High Energy Phys. 03 (2010) 130.

[48] S. Giusto, R. Russo, and D. Turton, New D1-D5-P geometries from string amplitudes, J. High Energy Phys. 11 (2011) 062.

[49] M. Bianchi, J.F. Morales, and L. Pieri, Stringy origin of $4 \mathrm{~d}$ black hole microstates, J. High Energy Phys. 06 (2016) 003.

[50] M. Bianchi, D. Consoli, A. Grillo, J. F. Morales, P. Pani, and G. Raposo, The multipolar structure of fuzzballs, arXiv: 2008.01445.

[51] K. Thorne, Multipole expansions of gravitational radiation, Rev. Mod. Phys. 52, 299 (1980). 
[52] Y. Gursel, Multipole moments for stationary systems: The equivalence of the Geroch-Hansen formulation and the Thorne formulation, Gen. Relativ. Gravit. 15, 737 (1983).

[53] I. Bena and N. P. Warner, Black holes, black rings and their microstates, Lect. Notes Phys. 755, 1 (2008).

[54] G. Gibbons and N. Warner, Global structure of fivedimensional fuzzballs, Classical Quantum Gravity 31, 025016 (2014).

[55] B. Bates and F. Denef, Exact solutions for supersymmetric stationary black hole composites, J. High Energy Phys. 11 (2011) 127.

[56] E. T. Newman, R. Couch, K. Chinnapared, A. Exton, A. Prakash, and R. Torrence, Metric of a rotating, charged mass, J. Math. Phys. (N.Y.) 6, 918 (1965).

[57] T. P. Sotiriou and T. A. Apostolatos, Corrected multipole moments of axisymmetric electrovacuum spacetimes, Classical Quantum Gravity 21, 5727 (2004).

[58] K. Akiyama et al. (Event Horizon Telescope Collaboration), First M87 event horizon telescope results. I. The shadow of the supermassive black hole, Astrophys. J. 875, L1 (2019).

[59] L. Blanchet, Gravitational radiation from post-Newtonian sources and inspiralling compact binaries, Living Rev. Relativity 9, 4 (2006).

[60] N. V. Krishnendu, K. G. Arun, and C. K. Mishra, Testing the Binary Black Hole Nature of a Compact Binary Coalescence, Phys. Rev. Lett. 119, 091101 (2017).

[61] B. Abbott et al. (LIGO Scientific and Virgo Collaborations), GWTC-1: A Gravitational-Wave Transient Catalog of Compact Binary Mergers Observed by LIGO and Virgo during the First and Second Observing Runs, Phys. Rev. X 9, 031040 (2019).

[62] B. Abbott et al. (LIGO Scientific and Virgo Collaborations), Tests of general relativity with the binary black hole signals from the LIGO-Virgo Catalog GWTC-1, Phys. Rev. D 100, 104036 (2019).

[63] F. Di Pasqua, A. Maselli, and P. Pani, Testing the Kerr hypothesis: Electromagnetic versus gravitational-wave constraints of the quadrupole moment (to be published).

[64] N. V. Krishnendu, C. K. Mishra, and K. G. Arun, Spininduced deformations and tests of binary black hole nature using third-generation detectors, Phys. Rev. D 99, 064008 (2019).

[65] P. Amaro-Seoane et al. (LISA Collaboration), Laser interferometer space antenna, arXiv:1702.00786.

[66] S. Babak, J. Gair, A. Sesana, E. Barausse, C. F. Sopuerta, C. P. L. Berry, E. Berti, P. Amaro-Seoane, A. Petiteau, and A. Klein, Science with the space-based interferometer LISA. V: Extreme mass-ratio inspirals, Phys. Rev. D 95, 103012 (2017).

[67] A. J. Chua, S. Hee, W. J. Handley, E. Higson, C. J. Moore, J. R. Gair, M. P. Hobson, and A. N. Lasenby, Towards a framework for testing general relativity with extreme-massratio-inspiral observations, Mon. Not. R. Astron. Soc. 478, 28 (2018).

[68] A. J. K. Chua, N. Korsakova, C. J. Moore, J. R. Gair, and S. Babak, Gaussian processes for the interpolation and marginalization of waveform error in extreme-mass-ratioinspiral parameter estimation, Phys. Rev. D 101, 044027 (2020).

[69] LISA Data Challenge Working Group. LISA Data Challenges, 2019, https://lisa-ldc.lal.in2p3.fr.

[70] L. Barack and C. Cutler, Using LISA EMRI sources to test off-Kerr deviations in the geometry of massive black holes, Phys. Rev. D 75, 042003 (2007).

[71] K. Glampedakis and S. Babak, Mapping spacetimes with LISA: Inspiral of a test-body in a "quasi-Kerr" field, Classical Quantum Gravity 23, 4167 (2006).

[72] K. Destounis, A. G. Suvorov, and K. D. Kokkotas, Testing spacetime symmetry through gravitational waves from extreme-mass-ratio inspirals, Phys. Rev. D 102, 064041 (2020).

[73] F. D. Ryan, Gravitational waves from the inspiral of a compact object into a massive, axisymmetric body with arbitrary multipole moments, Phys. Rev. D 52, 5707 (1995).

[74] I. Bena and D. R. Mayerson, following Letter, A New Window into Black Holes Phys. Rev. Lett. 125, 221602 (2020).

[75] I. Bena and D. R. Mayerson, Black holes lessons from multipole ratios, arXiv:2007.09152. 